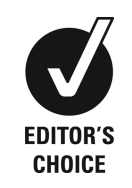
Endocrinology and General Medicine, University Hospital of North Midlands NHS Trust, Stoke on Trent, UK

\section{Correspondence to} Dr Ananth U Nayak, ananth.nayak@nhs.net

Accepted 19 December 2014

\title{
Splenic abscess as a potential initial manifestation of quiescent infective endocarditis in a patient with bronchopneumonia
}

\author{
Lindsay McOwat, Kah Fai Wong, George I Varughese, Ananth U Nayak
}

\section{SUMMARY}

A 78-year-old woman presented to the acute medical unit with a productive cough, dyspnoea and decreased appetite of 4 days duration. Initial assessment supported a diagnosis of right-sided community-acquired pneumonia and she was started on antibiotics. In view of the clinical finding of splenomegaly, she had an ultrasound and, subsequently, a CT of the abdomen, which revealed a large splenic abscess. Pending cultures from a sample obtained from percutaneous drainage of the abscess, she was started on intravenous meropenem. The initial echocardiogram did not suggest any evidence of endocarditis. The pus drained from the abscess on cultures was subsequently positive for Staphylococcus aureus. An MRI of the spine excluded discitis as a source of infection. Owing to a high index of clinical suspicion a repeat echocardiogram was undertaken after 1-week, which confirmed acute endocarditis. The patient was treated with intravenous antibiotics for 6 weeks with improvement in clinical, radiological and biochemical parameters.

\section{BACKGROUND}

Splenic abscess is a rare clinical entity that is usually secondary to haematogenous spread from an infective focus elsewhere in the body. Infective endocarditis (IE) can be associated with systemic embolisation and is associated with about $10 \%$ of reported incidence of splenic abscess. We report an interesting case of splenic abscess with subsequent evidence of Staphylococcal IE in an immunocompromised patient on repeat echocardiogram. Although IE is well recognised to be a source of infection when patients are investigated for abscesses elsewhere, a repeat echocardiogram is not usually considered in routine clinical practice especially when a patient has another infection, such as pneumonia, as in the index patient being reported.

\section{CASE PRESENTATION}

CrossMark

To cite: McOwat $L$,

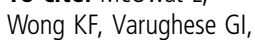
et al. BMJ Case Rep Published online: [please include Day Month Year] doi:10.1136/bcr-2014206794
A 78-year-old woman presented with productive cough, shortness of breath and decreased appetite for 4 days. She had been on long-term prednisolone for chronic inflammatory demyelinating polyneuropathy and had a history of pulmonary embolism and deep vein thrombosis.

Initial clinical assessment in the admission unit revealed signs of consolidation in the right lower lobe. She had normal heart sounds, a palpable but non-tender spleen and was febrile.

\section{INVESTIGATIONS}

The chest X-ray on admission supported the clinical diagnosis of consolidation in the right middle and lower lobe. Full blood count revealed a markedly raised white cell count of $90.4 \times 10^{9} / \mathrm{L}$ $\left(4.0-11 \times 10^{9} / \mathrm{L}\right)$ and neutrophilia of $84.9 \times 10^{9} / \mathrm{L}$ $\left(2.0-7.5 \times 10^{9} / \mathrm{L}\right)$. Peripheral blood film confirmed neutrophilia and thrombocytosis. This finding was persistent on repeated samples. The patient was treated for pneumonia with intravenous antibiotics as per hospital guidelines.

The abdominal ultrasound and subsequent CT imaging undertaken showed a large splenic abscess measuring $9.6 \times 8.2 \times 7.9 \mathrm{~cm}$ (figure 1 ). The patient was deemed unsuitable for splenectomy due to her comorbidities and was treated with broad-spectrum antibiotics (intravenous meropenem). Ultrasound guided percutaneous drainage of the abscess was performed successfully. Transthoracic echocardiogram (TTE) was undertaken to look for infective endocarditis as a source of the abscess; however, it did not reveal any vegetations attached to the cardiac valves and was reported as low suspicion of infective endocarditis.

The aspirate from the abscess cultured Staphylococcus aureus was sensitive to flucloxacillin, tetracycline and clarythromicin. A repeat ultrasound of the abdomen showed a sizeable splenic abscess despite drain in-situ. An MRI of the spine excluded discitis. A repeat TTE a week after the initial TTE revealed a small mobile mass on the posterior leaflet of the mitral valve, highly suggestive of infective endocarditis.

\section{TREATMENT}

The intravenous meropenem was changed to highdose intravenous flucloxacillin based on sensitivities and hospital guidelines for management of IE. It was methicillin-sensitive staphylococcus aureus (MSSA), hence the patient did not decompensate with meropenem. While the splenic drain remained in situ, the patient was transferred to the cardiology ward for management of her endocarditis, where she received a full 6 weeks course of antibiotics.

\section{OUTCOME AND FOLLOW-UP}

Repeat ultrasound a month after splenic drain insertion showed resolving collection. The patient had serial TTEs during the treatment course, which demonstrated continuing reduction in the mass size on the valvular leaflet. The echocardiogram on treatment completion showed only trivially 


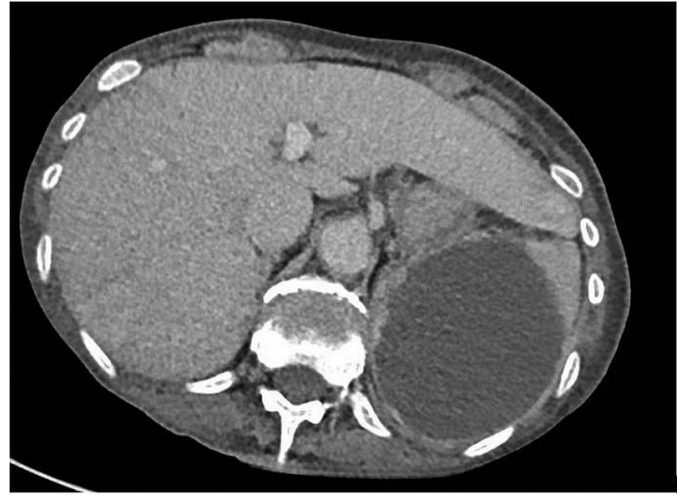

Figure $1 \mathrm{CT}$ of the abdomen revealing the large new low attenuation focus in the spleen representing an encapsulated splenic abscess.

thickened leaflets. Biochemical inflammatory markers improved significantly and were in normal range prior to the patient's discharge from hospital.

\section{DISCUSSION}

A splenic abscess, though rare (frequency $0.05-0.7 \%$ ), has a high mortality rate when untreated (almost 100\%), ${ }^{1}$ which can be lowered if the condition is appropriately managed. The classical presentation is with left upper quadrant abdominal pain, fever and splenomegaly. Imaging is necessary to facilitate diagnosis, common modalities being ultrasound and CT with abdominal ultrasound showing almost characteristic hypo anechoic or nearly anechoic, ovoid or round areas in the spleen. $^{2}{ }^{3}$ CT of the abdomen and pelvis with contrast can be more efficient in assessing the abscess and is sometimes the preferred modality. ${ }^{4}$

Splenic abscesses usually result due to embolisation from septic focus elsewhere, ${ }^{5}$ and localisation of the primary focus can be a challenge, as in our patient, who was immunocompromised being on long-term steroids. The initial TTE was normal and the IE was picked up on subsequent TTE undertaken because of clinical suspicion. Whether IE was the primary focus or secondary to a primary splenic pathology and abscess would be difficult to ascertain in this case. Limitations of TTE (compared to transoesophageal echocardiogram) would favour the former, though a potential splenic infarction resulting from haematological disorder could have led to secondary infection and abscess formation; this has also been reported. ${ }^{5}$

Diagnostic percutaneous aspiration guided by ultrasonography or CT scanning is useful in helping to confirm the diagnosis of splenic abscess and in providing a specimen for bacteriology. The medical management includes antibiotic therapy, which is usual based on sensitivities. Early diagnosis and percutaneous drainage is now considered an effective and relatively less invasive treatment option than surgical intervention with splenectomy. Percutaneous drainage can increase the chance of preserving the spleen and its immunological function, thus reducing the risk of overwhelming postsplenectomy infections. $^{2}{ }^{6}$ Surgery is reserved for patients who are stable and not amenable to percutaneous drainage. With patients in whom the less invasive treatment fails, laparoscopic splenectomy is now considered the most reliable treatment for this condition. ${ }^{2}$

\section{Learning points}

- Splenic abscess is rare but has a high mortality if untreated.

- When present, it is usually associated with primary focus elsewhere and commonly with infective endocarditis.

- Clinicians should have a low threshold to undertake more sensitive imaging for endocarditis such as transoesophageal echocardiogram, or they should undertake serial transthoracic echocardiograms when no other focus is identified.

- Treatment includes antibiotics based on sensitivity, image guided aspiration and, potentially, laparoscopic splenectomy in those who do not respond to the less invasive treatment.

Acknowledgements The authors would like to acknowledge the support from $\mathrm{Dr}$ Anthony Cadwgan, Consultant Physician in Infectious Diseases, for his input in the management of this patient.

Contributors LM and KFW contributed in the writing the initial draft of the manuscript. AUN and GIV contributed in the critical revision of the manuscript. All authors gave final approval of the manuscript.

Competing interests None.

\section{Patient consent Obtained.}

Provenance and peer review Not commissioned; externally peer reviewed.

\section{REFERENCES}

1 Cheesbrough JS, Jones EW, Finch RG. The management of splenic abscess. Q J Med 1985;57:653-7.

2 Fotiadis C, Lavranos G, Patapis $P$, et al. Abscesses of the spleen: report of three cases. World J Gastroenterol 2008;14:3088-91.

3 Chang FY, MacDonald BB, Peacock JE Jr, et al. A prospective multicenter study of Staphylococcus aureus bacteremia: incidence of endocarditis, risk factors for mortality, and clinical impact of methicillin resistance. Medicine (Baltimore) 2003;82:322-32

4 Chang KC, Chuah SK, Changchien CS, et al. Clinical characteristics and prognostic factors of splenic abscess: a review of 67 cases in a single medical center of Taiwan. World J Gastroenterol 2006;14:460-4.

5 Bayer AS, Bolger AF, Taubert KA, et al. Diagnosis and management of infective endocarditis and its complications. Circulation 1998;98:2936-48.

6 Faruque AV, Qazi SH, Arshad M, et al. Isolated splenic abscess in children, role of splenic preservation. Pediatr Surg Int 2013;29:787-90. 